\title{
Research on Multifunctional Glove for Electrical Work
}

\author{
Pang Yanjun, Wang Zhaoxia, Cheng Xingjun, Wang Liu, Hai Tianshu, Li Xinyu, Li \\ Bo, Wang Chuang, Yue Yangzhuo, Chen Fengjun, Liu Min, Zhang Hua \\ Fushun Power Supply Company, Liaoning Electric Power Company Limited, State Grid, China
}

fushunpowersupply@163.com

Keywords: electric measurement; safety apparatus; multifunctional glove; electrical work

\begin{abstract}
This paper introduces several common shortcomings of work gloves in electric energy measurement, and focuses on the disadvantages of the spandex gloves; it expounds the method and technique principle of multifunctional gloves for electrical work and introduces the main parameters and field application effect of the gloves. Therefore, it is concluded that the multifunctional glove for electrical work not only solve the security problems, but also saves time and effort, greatly improving the work efficiency, and can strengthen the quality and quantity of the calibration task for the company.
\end{abstract}

\section{Introduction}

Electric energy measurement is a highly specialized field, which has very strict requirements for measuring instruments and safety equipment. According to the requirements of SGCC, Fushun power supply company began to replace a large number of single phase cost control intelligent electric energy meter from 2009,and the calibration personnel in electric energy measurement center is to small to complete the large quantities of calibration task, so it must improve work efficiency, reduce the safety hidden trouble. Now we use spandex glove to check electric energy measurement, although spandex gloves have good elasticity and permeability, but too smooth to avoid slipping in carrying and dismounting electric energy meter, thus exist security risks such as a broken meter or injured personnel check phenomenon, In addition, its fingertips is very thin, and has poor abrasion resistance, the tips of gloves is easy to grind away when sealing, then hurt the hand and influence work efficiency. Hence one can see that spandex gloves are not suitable for checking electric energy meter. Therefore, we developed the power multifunctional gloves to solve existing problems, to ensure timely completion of verification tasks[1-3].

\section{Several frequently-used work gloves}

(1)Cotton yarn gloves are made by cotton fiber weaving machines, this glove's flexibility and permeability is not good, its shape can not change with hands, easy to scratch on the steel line of the seal at power meter checking works, and affects the working efficiency, so we do not use cotton gloves.

(2)Dotted gloves use special anti-static polyester cloth, these gloves is anti-skidding anti - static and breathable, but its wear resistance is not good, due to the long time of the fingers, the finger parts will wear out quickly, it is not safe for check operator's fingers, so we do not use dotted gloves[4, 5].

(3)PU coating gloves are made of knitted nylon, its fingers or palm is coated by PU resin. It is anti-friction, anti-skidding, corrosion preventive, but very loose, hypertrophy, thick, and has poor air permeability, causes sweating in the heat after wearing a long time, this Glovers affect the efficiency, so we do not use PU coated gloves.

(4)Spandex gloves are made of polyester or nylon fiber, it is high elasticity, good air permeability, comfortable and not deformation when be pulled, but spandex gloves have poor abrasion performance, and too smooth to avoid slipping in carrying and dismounting electric energy meter, it is easy to hurt check operator; it also affects the efficiency owing to Sore fingers, so this kind of gloves is not suitable. 


\section{Disadvantages of spandex gloves in use}

Through many years of work experience and summary, we analyze and discuss the spandex glove continuously, and then obtained the following drawbacks:

(1)Poor abrasion performance, The concentrated force puts on the finger in daily seal work, this way causes the damage of the finger part of the glove easily. It consumes on average a spandex glove per person per day as the working intensity nowadays.

(2)Good air permeability, spandex gloves are too smooth to avoid slipping in carrying and dismounting electric energy meter, the meter often slide from hands to be broken and also hurt check operator

(3)Poor air permeability, the spandex glove causes sweating in the heat after wearing a long time in summer, then affect the efficiency.

(4)No anti-static function, the precision electronic components in the electric energy meter can be easily damaged by static electricity.

(5)No insulation performance, we use the spandex gloves are not insulated, it is easy to happen electric accidents if both hands with the voltage clamp at the same time, affects the whole power system, brings great loss to the company, and causes great harm to the family.

(6) High cost, each pair of spandex glove is $3 \mathrm{RMB}$; it consumed on average 264 pairs of gloves per month, and consumed gloves funds on average 9504 RMB per year.

\section{Method for developing multifunctional gloves for electric power work}

According to the shortcomings of the spandex gloves, we combine the advantage of several kinds of labor insurance gloves, and decided to select again, then developed a new electricity multifunctional gloves. the Method for developing multifunctional gloves for electric power work is built the PU resin up of thumb, index finger, middle finger on the basis of dotted gloves, it makes this gloves with good abrasion resistance, skid resistance, anti-static, scalability and permeability, etc., refrain from abasing the finger parts for a long time work avoids hurting check operator due to the meter slipping in carrying and dismounting electric energy meter, solves the problem of sweating in the heat after wearing a long time reduces the damage of the electronic components caused by the electrostatic also prevents check operator from electric accidents. Development of the kind of gloves not only reduces the safety hazards, but also improves the work efficiency; ensure the completion of large quantities of calibration tasks.

\section{Technology principle of multifunctional glove for electrical work}

The technology principle of multifunctional glove for electrical work is based on the material of elastic, air permeability and antistatic property, prevent the glove loose , keep the precision work away from the unsuitable heat for ,avoid the damage to the electronic components, and also doing PVC point processing on stress section of the palm to increase friction, avert the damage of the meter from carrying and dismounting, even fall hurt. Built the PU resin up of fingers, reduce the damage caused by the sealing line to the finger. Significantly decrease the frequency of replacement gloves to save labor costs. The principle design of multifunctional glove for electrical work is Figure 1.

\section{Main parameters of multifunctional glove for electrical work}

(1)Dotted PVC is a kind of polymer material, which is a kind of non-crystalline material. PVC materials s often added stabilizer, lubricant, auxiliary processing agent, pigment, anti impact modifier and other additives in practical use. It Is non-inflammability, high strength, excellent resistance to climate variability and Excellent geometrical stability. PVC had strong resistance to The oxidizing agent, reducing agent and acid. It is characterized by mechanical properties, good electrical properties, strong acid and alkali resistance, good chemical stability, but low softening 
point. Its parameters are: $1.38 \mathrm{~g} / \mathrm{m} 3$, forming shrinkage rate: $0.6-1.5 \%$, forming temperature: 160 $190^{\circ} \mathrm{C}$.

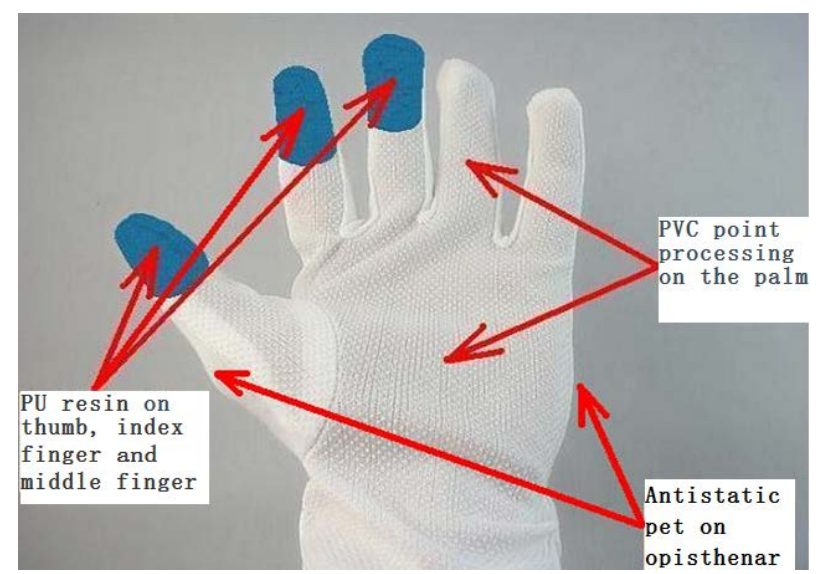

Fig.1 the principle drawings of multifunctional glove for electrical work

(2) The Chinese name of PU coated is polyurethane, it has good over-all properties, hardness, fullness, adhesion, durability, hand handle are also good. Its parameters are Atomization enthalpy: $\mathrm{kJ} / \mathrm{mol}$ at $25^{\circ} \mathrm{C} 360$; thermal conductivity: w/(M • K) 674; heat of vaporization: $(\mathrm{kJ} / \mathrm{mol}) 344.0$; electrical conductivity : $10^{6} /(\mathrm{cm} \bullet \Omega) 0.00666$; heat of fusion $(\mathrm{kJ} / \mathrm{mol}) 2.840$; heat capacity’s/(mol K)35.5.

(3) Polyester is a synthetic fiber, which is the name of our country's polyester fiber. It is a fiber obtained by spinning and polyethylene glycol terephthalate (PET), which is a kind of fiber polymer made from diethyl terephthalate (PTA) or dimethyl terephthalate (DMT) and ethylene glycol (EG) through etherification or ester exchange and condensation reaction. The parameters: short fiber strength is $2.6 \sim 5.7 \mathrm{cN} / \mathrm{dtex}$; high strength fiber is $5.6 \sim 8.0 \mathrm{cN} / \mathrm{dtex}$; it has good elasticity, and can be completely recovered when the elongation is $5 \% \sim 6 \%$. Heat resistance and thermal stability are the best in synthetic fiber fabrics. The surface of the polyester is smooth and the inner molecules are arranged closely. It has good abrasion performance, Polyester has the second-best abrasion performance after nylon, it is better than other natural and synthetic fibers. It has good light fastness, Polyester has the second-best light fastness after acrylic fibers, it is better than other natural and synthetic fibers. Corrosion resistance can resistance to bleaching agent, oxidizing agent, hydrocarbons, ketenes petroleum products and inorganic acid. Alkali resistant, it is not afraid of mold, but hot alkali can make it decomposed. Good color fastness, not easy to fade.

(4) Conductive fiber is a new kind of fiber, which has good electrical conductivity and durability, especially in low humidity, still has good durability, so it has a great application in many fields.

\section{Application effect of multifunctional glove for electrical work}

(1)Application

Calibration classes began to use the multifunctional glove in 2010.the calibration staff have been completed in the tens of thousands of single phase charge meter calibration work with this gloves, in general, the performance of the multifunctional glove is all that could be desired. It is widely recognized in terms of good air permeability, good antistatic ability, good insulation performance, high elasticity, anti-abrasion and anti-skidding capability, high working efficiency. The use of the multifunctional glove saves the cost, avoids the loss, and greatly improves the work efficiency, also lays a good foundation for the quality and quantity of the calibration task. The Figure 2 is the impression drawing of the multifunctional glove for electrical work. 

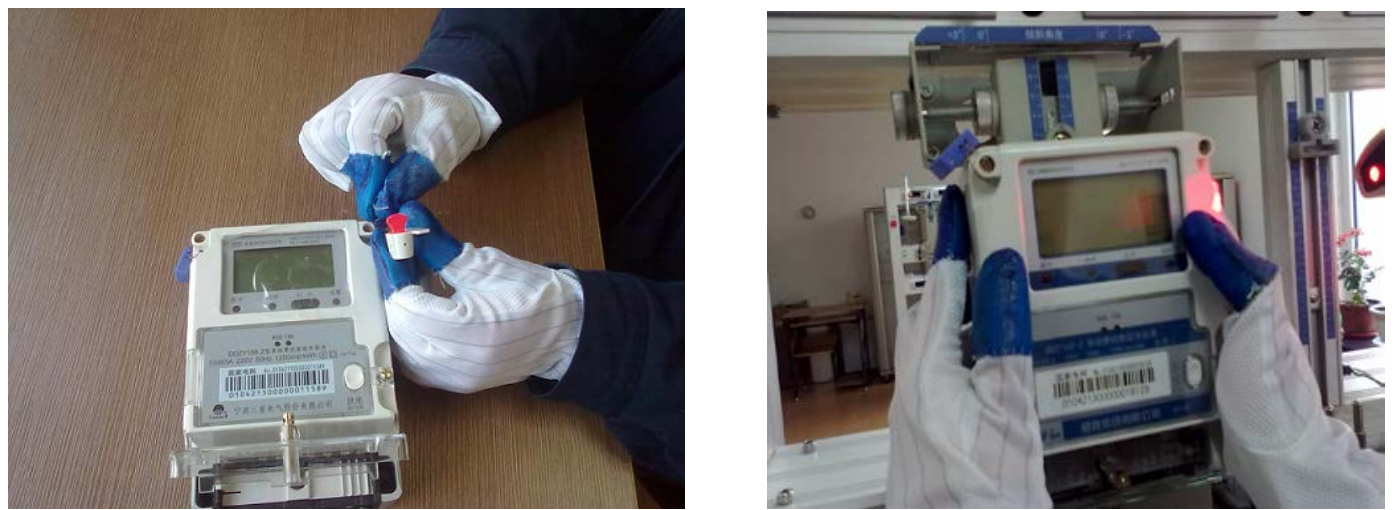

Fig.2 The impression drawing of the multifunctional glove for electrical work

(2)Benefit analysis

The benefits are very significant when the project is put into use:

1)It does not need to replace the gloves, saves money 8064 RMB per year

2)It avoids the sliding phenomenon of the electric energy meter, saves $3600 \mathrm{RMB}$ per year.

3)It protects the personal safety, saves $1000 \mathrm{RMB}$ per year..

4)It has good insulation, avoids the occurrence of electric shock accident, reduces the security risks, and provides security for work.

\section{Conclusion}

(1) The palm of the glove has the plastic part, that makes it with the anti-abrasion and antiskidding capability when handling and dismounting the electricity meters, the glove is flexible operation and it has the high flexibility.

(2) The opisthenar of the glove is made of special anti-static polyester cloth, which can avoid the damage caused by the static electricity. The glove has good flexibility and air permeability; the inspection staff will not feel hot when doing a long time operation,

(3) The glove is safe and reliable. It can avoid broken and hurting check operator due to the meter slipping in carrying and dismounting electricity meter, also it does not occur the appearance that the seal scratches finger; avoids the loss of property and personnel injuries accidents.

(4) The PU coated of good insulation lying on the glove's fingers avoid personal electric shock accident.

(5) The PU coated of good insulation lying on the glove's fingers avoid personal electric shock accident.

To sum up, the development and application of multifunctional glove for electrical work not only solves the security problems, but also saves time and effort, greatly improve the work efficiency, and can strengthen the quality and quantity of the calibration task for the company.

\section{Reference}

[1] Selection Rules for Labor Protection Articles, GB11651-1989

[2] The Classification Standard of Work Gloves, Sep.25, 2007.

[3] The Selection and Use Standard of Work Gloves, GB11651-1989

[4] General Technical Specification for Insulated Gloves in Live working, GB17622-1998

[5] Safe Use of Insulated Gloves, GB/T16927.1-1997 